\title{
Risk of cardiovascular disease among patients with sarcoidosis: a population-based retrospective cohort study, 1976-2013
}

\author{
Patompong Ungprasert ${ }^{1}$, Cynthia S. Crowson ${ }^{1,2}$ and Eric L. Matteson ${ }^{1,3}$ \\ Affiliations: ${ }^{1}$ Division of Rheumatology, Dept of Internal Medicine, Mayo Clinic, Rochester, MN, USA. ${ }^{2}$ Division \\ of Biomedical Statistics and Informatics, Dept of Health Science Research, Mayo Clinic, Rochester, MN, USA. \\ ${ }^{3}$ Division of Epidemiology, Dept of Health Science Research, Mayo Clinic, Rochester, MN, USA.
}

Correspondence: Patompong Ungprasert, Division of Rheumatology, Dept of Internal Medicine, Mayo Clinic, 200 First Avenue SW, Rochester, MN 55905, USA. E-mail: Ungprasert.Patompongamayo.edu

@ERSpublications

Patients with sarcoidosis have a higher risk of cardiovascular diseases http://ow.ly/EuTe306jcYW

Cite this article as: Ungprasert P, Crowson CS, Matteson EL. Risk of cardiovascular disease among patients with sarcoidosis: a population-based retrospective cohort study, 1976-2013. Eur Respir J 2017; 49: 1601290 [https://doi.org/10.1183/13993003.01290-2016].

ABSTRACT A higher incidence of cardiovascular disease (CVD) has been observed in several chronic inflammatory diseases. However, data on sarcoidosis are limited.

In this study, 345 patients with incident sarcoidosis in Olmsted County (Minnesota, USA) during 1976-2013 were identified based on comprehensive medical record review. 345 sex- and age-matched comparators were also identified from the same underlying population. Medical records were individually reviewed for $\mathrm{CVD}$, including coronary artery disease, congestive heart failure, atrial fibrillation, cerebrovascular accident, transient ischaemic attack, peripheral arterial disease and abdominal aortic aneurysm. Cox proportional hazards models with adjustment for age, sex, calendar year and cardiovascular risk factors were used to compare the rate of development of CVD between cases and comparators.

The prevalence of CVD before the index date was not significantly different between the two groups. Adjusting for age, sex and calendar year, the risk of incident CVD after the index date was significantly elevated among patients with sarcoidosis with an adjusted hazard ratio of 1.57 (95\% CI 1.15-2.16). Adjustment for cardiovascular risk factors yielded an adjusted hazard ratio of 1.65 (95\% CI 1.08-2.53). Significantly increased risk was also observed for several types of CVD, including coronary artery disease, congestive heart failure, atrial fibrillation and cerebrovascular accident.

Increased incidence of CVD among patients with sarcoidosis was demonstrated in this population-based cohort, even after controlling for baseline traditional atherosclerotic risk factors.

Received: June 292016 | Accepted after revision: Nov 112016

Support statement: This study was made possible using the resources of the Rochester Epidemiology Project, which is supported by the National Institute on Aging of the National Institutes of Health (NIH) under award number R01AG034676, and CTSA grant number UL1 TR000135 from the National Center for Advancing Translational Sciences (NCATS), a component of the NIH. Funding information for this article has been deposited with the Open Funder Registry. The content is solely the responsibility of the authors and does not necessarily represent the official views of the NIH.

Conflict of interest: None declared.

Copyright OERS 2017 


\section{Introduction}

Cardiovascular disease (CVD) is one of the leading causes of morbidity and mortality worldwide. In the USA, it is responsible for $17 \%$ of national healthcare expenditure [1]. Traditional risk factors for CVD include diabetes mellitus, obesity, hypertension, dyslipidaemia, age, sex, sedentary lifestyle and smoking [2].

Over the past decades, chronic inflammation has been increasingly recognised as a nontraditional risk factor for CVD as several studies have revealed a higher incidence of premature atherosclerosis among patients with chronic inflammatory diseases [3-5]. Oxidative stress and inflammatory cytokines are believed to be the cornerstone of the accelerated atherosclerosis as their deleterious effects on endothelial function have been consistently demonstrated $[6,7]$.

Sarcoidosis is an inflammatory disorder of unknown aetiology that can affect virtually any organ. The prevalence and clinical manifestations of sarcoidosis vary across different populations [8, 9]. Presence of noncaseating granuloma is the histopathological hallmark of the disease. The clinical course of sarcoidosis can range from an acute self-limited process to progressive organ dysfunction with significant morbidity and mortality.

Patients with sarcoidosis might also have a higher risk of CVD. A coding-based study from Sweden found an increased risk of coronary artery disease (CAD) among patients with sarcoidosis with a standardised incidence ratio of 1.15 (95\% CI 1.09-1.20). Nonetheless, the accuracy of the study was limited by the study design that used an administrative registry to identify the cohort and the outcome of interest without further case/event verification [10].

The current study aimed to better characterise the risk of CVD among patients with sarcoidosis using the previously identified cohort of patients with incident sarcoidosis in Olmsted County (Minnesota, USA) from 1976 to 2013. The incidence of CVD among patients with sarcoidosis was compared with the incidence of CVD among sex- and age-matched comparator subjects without sarcoidosis randomly selected from the same population.

\section{Methods}

Participants and study design

Through the resources of the Rochester Epidemiology Project, the population of Olmsted County is well suited for investigation of the epidemiology of sarcoidosis because comprehensive medical records for all residents seeking medical care for over six decades are available. The record linkage system allows ready access to the medical records, for inpatients and outpatients, from all healthcare providers for the local population, including the Mayo Clinic, the Olmsted Medical Center and their affiliated hospitals, local nursing homes, and the few private practitioners. The potential of this data system for use in population-based studies has previously been described [11]. This system ensures virtually complete ascertainment of all clinically recognised cases of sarcoidosis among the residents of Olmsted County. The system also allows complete ascertainment of the outcomes of interest (i.e. CVD) for cases and comparators.

The sarcoidosis cohort consists of 345 previously identified cases in Olmsted County, incident from 1976 to 2013 [12]. Potential cases were screened for sarcoidosis using diagnosis codes related to sarcoid, sarcoidosis and contextual noncaseating granuloma, and the medical records of all potential cases were individually reviewed. Inclusion required physician diagnosis supported by histopathology and radiological features of intrathoracic sarcoidosis, compatible clinical presentation, and exclusion of other granulomatous diseases such as tuberculosis. Tissue samples were considered positive if they demonstrated noncaseating granuloma without evidence of acid-fast bacilli or fungi. The only exception to the requirement of histopathological confirmation was stage I pulmonary sarcoidosis that required only radiographic evidence of symmetric bilateral hilar adenopathy in the absence of other identifiable causes. Isolated granulomatous disease of a specific organ except for the skin was also included if there was no better alternative diagnosis [13]. Cases with a diagnosis of sarcoidosis prior to residency in Olmsted County were not included.

For each sarcoidosis patient, one comparator without sarcoidosis at the time of the patient's sarcoidosis diagnosis was randomly selected and assigned an index date that corresponded to the sarcoidosis incidence date. Matching criteria were similar age $( \pm 3$ years $)$ and same sex.

The medical records of cases and comparators were then reviewed for cardiovascular events which included $\mathrm{CAD}$, congestive heart failure $(\mathrm{CHF})$, atrial fibrillation, cerebrovascular accident (CVA), transient ischaemic attack, peripheral arterial disease and abdominal aortic aneurysm. CAD included both nonfatal and fatal myocardial infarction. Classification was based on physician diagnosis from clinical notes supported by coronary angiography. Patients who underwent coronary revascularisation procedures (percutaneous transluminal coronary angioplasty and coronary artery bypass grafting) were also classified as CAD. CHF was classified based on the Framingham criteria for CHF [14]. Atrial fibrillation was classified based on an ECG. CVA was defined as ischaemic stroke, haemorrhagic stroke, subarachnoid haemorrhage or death from 
CVA. Classification required physician diagnosis verified by imaging studies or cerebrospinal fluid analysis. Transient ischaemic attack was classified based on physician diagnosis and negative brain imaging study. Peripheral arterial disease was classified based on a resting ankle-brachial systolic pressure index of $\leqslant 0.9$ [15]. Abdominal aortic aneurysm was classified based on imaging showing the diameter of the abdominal aorta more than 1.5 times normal diameter [16]. Data on baseline cardiovascular risk factors, including smoking status, body mass index (BMI), diabetes mellitus, hypertension and dyslipidaemia, were also collected. The 10-year general Framingham risk score for CVD was calculated and the office-based 10-year Framingham risk score, which does not include laboratory values, was used when lipid values were unavailable [17]. Data on treatment with disease-modifying antirheumatic agents (DMARDs), biological agents and glucocorticoids were also collected. Follow-up was continued until death, migration or January 1, 2015. The study was approved by the Institutional Review Board of Mayo Clinic and Olmsted Medical Center. The need for informed consent was waived.

\section{Statistical analysis}

Descriptive statistics (percentages, mean, etc.) were used to summarise the characteristics of each cohort. Comparisons between cohorts were performed using Chi-squared and rank sum tests. Comparisons of prior CVD overall and by type were compared between cohorts using Fisher's exact tests to examine whether there were any increases in prevalence of CVD prior to development of sarcoidosis. The cumulative incidence of CVD overall and by type adjusted for the competing risk of death was estimated [18]. These methods are similar to the Kaplan-Meier method with censoring of patients who are still alive at last follow-up. However, patients who die before experiencing CVD events are appropriately accounted for to avoid overestimation of the rate of occurrence of CVD events, which can occur if such subjects are simply censored at death. For each type of CVD, patients whose first occurrence was prior to the diagnosis of sarcoidosis, or prior to the index date for subjects in the nonsarcoidosis comparison cohort, were excluded from the analysis of cumulative incidence. For the estimation of the cumulative incidence of CVD overall, the date of occurrence was the earliest date of occurrence of any of the individual CVD events.

Cox proportional hazards models were used to compare the rate of development of CVD, individually and in combination, between patients with sarcoidosis and the nonsarcoidosis comparison cohort. In addition, Cox proportional hazards models were used to assess the association of risk factors on the development of CVD events among patients with sarcoidosis. Risk factors of interest included those described above. Only events affecting an adequate number of patients $(n \geqslant 20)$ were examined in these risk factor analyses. Additional analyses to investigate the association between treatment (DMARDs, biological agents or glucocorticoids) and CVD were also performed among patients with sarcoidosis. Treatment was modelled as a time-dependent covariate. A p-value of $<0.05$ was considered statistically significant for all analyses. Analyses were performed using SAS version 9.4 (SAS Institute, Cary, NC, USA) and R version 3.1.1 (R Foundation for Statistical Computing, Vienna, Austria).

\section{Results}

Baseline characteristics of cases and comparators are described in table 1. Mean length of follow-up was 15.1 and 16.8 years for cases and comparators, respectively, corresponding to 5210 and 5863 total person-years of observation, respectively. There were more African-Americans among cases, while there were more current smokers among comparators. Mean BMI of cases was higher than comparators, but there was no difference in prevalence of other cardiovascular risk factors.

The prevalence of overall CVD prior to date of diagnosis/index date was not significantly different between patients with sarcoidosis and the nonsarcoidosis comparison cohort (3.8\% and $6.1 \%$, respectively; $\mathrm{p}=0.22$ ). Cumulative incidence of CVD after diagnosis/index date for cases and comparators is shown in figure 1. The risk of incident CVD adjusted for age, sex and calendar year was significantly higher among patients with sarcoidosis with a hazard ratio (HR) of 1.57 (95\% CI 1.15-2.16). Significantly elevated risk was observed in several subtypes of CVD, including CAD, CHF, atrial fibrillation and CVA. Further adjustment for current smoker, obesity, diabetes mellitus, hypertension and dyslipidaemia slightly increased the HR of overall CVD to 1.65 (95\% CI 1.08-2.53).

To reduce the likelihood of surveillance bias, sensitivity analysis including only CVD events that occurred at least 6 months after date of diagnosis/index date was performed. This resulted in exclusion of five overall CVD events among the cases and no events among the comparators. The HR for overall CVD from this sensitivity analysis adjusted for age, sex and calendar year was 1.50 (95\% CI 1.09-2.06). Significantly elevated risk was observed in CAD, CHF, atrial fibrillation and CVA, similar to the complete analysis. The numbers of events, cumulative incidence at 10 years and HR values of individual CVDs comparing patients with sarcoidosis with subjects without sarcoidosis adjusted for demographics and demographics plus baseline cardiovascular risk factors are demonstrated in table 2. 


\begin{tabular}{|c|c|c|c|}
\hline & Cases & Comparators & p-value \\
\hline Subjects & 345 & 345 & \\
\hline Age years & $45.6 \pm 13.6$ & $45.4 \pm 13.7$ & 0.87 \\
\hline Female & $174(50)$ & $174(50)$ & 1.0 \\
\hline Ethnicity & & & $<0.001$ \\
\hline Caucasian & $301(90)$ & 327 (95) & \\
\hline African-American & $18(5)$ & $2(1)$ & \\
\hline Asian & $6(2)$ & $0(0)$ & \\
\hline Native-American & $2(1)$ & $0(0)$ & \\
\hline Other & $7(2)$ & $14(4)$ & \\
\hline Unknown & 11 & 2 & \\
\hline Length of follow-up years & $15.1 \pm 10.5$ & $16.8 \pm 10.8$ & \\
\hline Smoking status & & & $<0.001$ \\
\hline Never & $198(60)$ & 132 (42) & \\
\hline Ex-smoker & $71(21)$ & 70 (22) & \\
\hline Current smoker & $63(19)$ & $115(36)$ & \\
\hline Unknown & 13 & 28 & \\
\hline BMI $\mathrm{kg} \cdot \mathrm{m}^{-2}$ & $29.8 \pm 7.3$ & $27.5 \pm 5.6$ & $<0.001$ \\
\hline Hypertension & 75 (22) & 75 (22) & 1.0 \\
\hline Dyslipidaemia & $50(14)$ & $57(17)$ & 0.46 \\
\hline Diabetes mellitus & $31(9)$ & $26(8)$ & 0.49 \\
\hline Framingham risk score & $8.5 \pm 9.0$ & $9.3 \pm 11.2$ & 0.78 \\
\hline
\end{tabular}

Data are presented as $\mathrm{n}$, mean \pm SD or $\mathrm{n}(\%)$, unless otherwise stated. BMI: body mass index.

Additional analyses to compare the risk of CVD, individually and in combination, between patients with sarcoidosis who had lower and higher inflammatory burden were performed. The requirement to use immunosuppressive therapy (DMARDs, biological agents or glucocorticoids) was used as the surrogate for higher inflammatory burden. Treatment was modelled as a time-dependent covariate. 151 patients (44\%) required immunosuppressive therapy at some point during follow-up. The HR for overall CVD between those who required and did not require immunosuppressive therapy adjusted for age, sex and calendar year was 1.25 (95\% CI 0.83-1.90). HR values of individual CVDs were also not statistically significant, as demonstrated in table 3.

\section{Discussion}

Over the past few decades, mortality from organ damage and other complications directly related to disease pathology has been declining in patients with immune-mediated diseases because of advances in therapy attributable to the better understanding of disease pathogenesis. As a result, those patients live longer, and CVD has emerged as a major cause of morbidity and mortality [19].

The current study is the first population-based study with a comprehensive medical record review to demonstrate the increased incidence of CVD among patients with sarcoidosis. Increased risk was observed

FIGURE 1 Cumulative incidence of overall cardiovascular disease among patients with sarcoidosis and comparators without sarcoidosis.

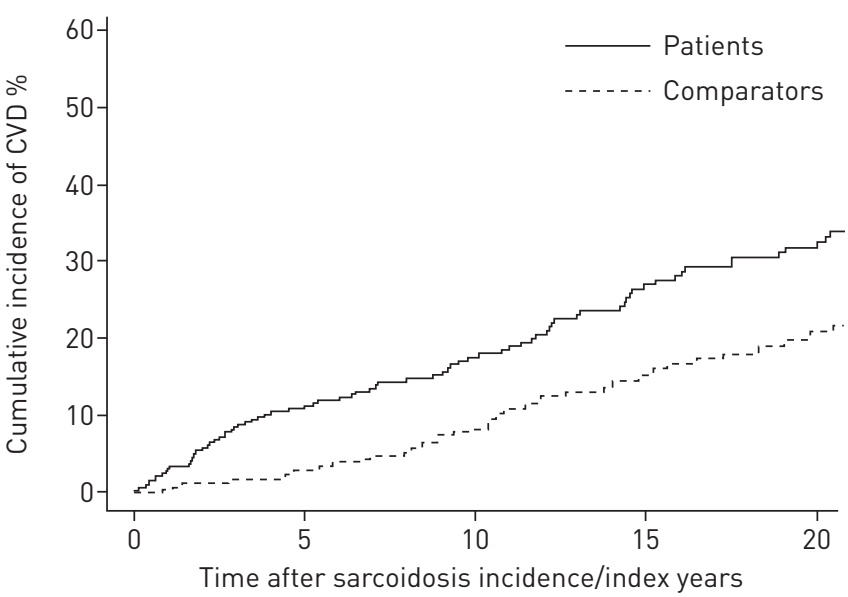


TABLE 2 Numbers of events and hazard ratio (HR) of individual cardiovascular diseases (CVDs) comparing patients with sarcoidosis with subjects without sarcoidosis

\begin{tabular}{|c|c|c|c|c|c|}
\hline & \multirow[t]{2}{*}{$\begin{array}{c}\text { Events after index } \\
\text { date for cases/comparators }\end{array}$} & \multicolumn{2}{|c|}{$\begin{array}{l}\text { Cumulative incidence } \\
(95 \% \mathrm{CI}) \text { at } 10 \text { years }\end{array}$} & \multirow[t]{2}{*}{$\operatorname{HR}(95 \% \mathrm{CI})^{\#}$} & \multirow[t]{2}{*}{$\operatorname{HR}(95 \% \mathrm{CI})^{\uparrow}$} \\
\hline & & Cases & Comparators & & \\
\hline Coronary artery disease & $54 / 38$ & $8.3(5.0-11.5)$ & $5.3(2.6-7.9)$ & $1.55(1.02-2.35)$ & $1.58(0.89-2.80)$ \\
\hline Congestive heart failure & $45 / 24$ & $7.6(4.5-10.7)$ & $1.8(0.2-3.4)$ & $2.06(1.25-3.38)$ & $2.72(1.46-5.06)$ \\
\hline Atrial fibrillation & $33 / 18$ & $4.6(2.0-7.1)$ & $2.2(0.4-4.0)$ & $1.93(1.08-3.43)$ & $1.90(0.97-3.93)$ \\
\hline Cerebrovascular accident & $32 / 14$ & $5.2(2.6-7.7)$ & $0.6(0.0-1.4)$ & $2.51(1.34-4.71)$ & $3.32(1.53-7.18)$ \\
\hline Abdominal aortic aneurysm & $4 / 4$ & $1.0(0.0-2.1)$ & $0.6(0.0-1.5)$ & $1.13(0.28-4.53)$ & $1.83(0.26-12.60)$ \\
\hline Any CVD & $96 / 65$ & $17.6(12.9-22.0)$ & $8.1(4.8-11.3)$ & $1.57(1.15-2.16)$ & $1.65(1.08-2.53)$ \\
\hline
\end{tabular}

Data are presented as $\mathrm{n} / \mathrm{n}$ or \%, unless otherwise stated. " : for all events after index date, adjusted for age, sex and calendar year; ${ }^{\natural}$ : for all events after index date, adjusted for age, sex, calendar year, current smoking, diabetes mellitus, hypertension, dyslipidaemia and obesity.

after adjusting for baseline cardiovascular risk factors and after excluding events that occurred within 6 months after date of diagnosis/index date in order to reduce the likelihood of surveillance bias.

Premature atherosclerosis associated with inflammation is probably the key driver behind this increased risk $[6,7]$. While not specifically studied in sarcoidosis, it is likely that several inflammatory cytokines, such as interleukin-1 and tumour necrosis factor, contribute to atherogenesis in this disease as well as other systemic inflammatory diseases through a number of mechanisms, including vascular adhesion molecule-1, leukocyte and matrix metalloproteinase activation [20,21]. Furthermore, chronic inflammation is known to promote initiation and propagation of the coagulation cascade, downregulate the anticoagulant pathway and inhibit fibrin removal $[22,23]$. Nonetheless, the additional analyses comparing patients with higher and lower disease activity using the requirement of immunosuppressive therapy as the surrogate failed to show a significantly different CVD risk between the two groups, which might suggest that other underlying mechanisms, in addition to the increased inflammatory burden, are also responsible for the elevated CVD risk.

It is conceivable that there is a shared genetic predisposition to both immune-mediated inflammatory disease and CVD. This shared genetic predisposition theory is well recognised in rheumatoid arthritis as its major risk gene, i.e. HLA-DRB1, is also associated with increased risk of CAD [24, 25]. HLA-DRB1 appears to predispose to development of rheumatoid arthritis by promoting the selection and survival of autoreactive $\mathrm{CD}^{+} \mathrm{T}$-cells which can be found in both synovial tissue and atherosclerotic plaque [26]. Interestingly, a large case-control study investigating possible aetiologies of sarcoidosis found that $D R B 1^{\star} 1101$ was significantly associated with increased risk of sarcoidosis in both African-Americans and Caucasians [27]. The data are preliminary and further investigations would be required to evaluate a possible shared genetic predisposition between sarcoidosis and CVD.

Glucocorticoids, the most commonly used medication in the management of sarcoidosis, may also be a contributing factor to the increased risk of CVD as their long-term use is associated with several traditional CVD risk factors, such as diabetes mellitus, hypertension and dyslipidaemia [28].

TABLE 3 Hazard ratio (HR) values for association between treatment lany disease-modifying antirheumatic drugs, biological agents and/or glucocorticoids) and individual cardiovascular diseases (CVDs) in 345 patients with sarcoidosis during 1976-2013

\begin{tabular}{lcc} 
& HR $\left(95 \% \mathbf{C l}^{\#}\right.$ & p-value \\
\hline Coronary artery disease & $1.25(0.72-2.18)$ & 0.43 \\
Congestive heart failure & $1.58(0.86-2.90)$ & 0.14 \\
Atrial fibrillation & $0.76(0.37-1.56)$ & 0.46 \\
Cerebrovascular accident & $0.92(0.45-1.89)$ & 0.83 \\
Transient ischaemic attack & $0.27(0.03-2.38)$ & 0.24 \\
Peripheral arterial disease & $2.22(0.71-6.93)$ & 0.17 \\
Abdominal aortic aneurysm & $0.51(0.05-5.49)$ & 0.58 \\
Any CVD & $1.25(0.83-1.90)$ & 0.29 \\
\hline
\end{tabular}

\# : adjusted for age, sex and calendar year of sarcoidosis incidence/index date. 
The major strengths of this study are that it is a population-based study using a comprehensive record linkage system that allows capture of nearly all the clinically recognised cases of sarcoidosis in the community. This approach minimises referral bias and allows capture of the complete spectrum of the disease. The diagnosis of sarcoidosis was verified by medical record and histopathology report review that minimised the likelihood of misclassification, a common concern in coding-based studies. The cohorts also had a long length of follow-up with the mean duration of follow-up $>15$ years. An adequate duration of follow-up is of particular importance for a CVD study as the events of interest might not occur until several years after the index date/date of diagnosis.

The major limitations are those inherent in the retrospective nature of the study. Clinical information of the cohorts was not systematically obtained and, thus, all the pertinent clinical data might not be recorded. Cardiac involvement of sarcoidosis could mimic signs and symptoms of some events of interest, including $\mathrm{CAD}, \mathrm{CHF}$ and atrial fibrillation. It was not possible to retrospectively interrogate a putative relationship of these events to cardiac sarcoidosis. Therefore, it is possible that there were misclassifications which could lead to falsely elevated incidence rates. The analyses did not adjust for several potential confounders such as depression, healthcare access and socioeconomic status. Surveillance bias may play a role as patients with sarcoidosis, because of their illness, might undergo more medical examinations and laboratory investigations. However, sensitivity analysis that included only cardiovascular events that occurred at least 6 months after the index date did not yield significantly different results. The results of this study might not be generalisable to other populations as the clinical phenotype and epidemiology of sarcoidosis vary among different racial groups [8], but the population of Olmsted County is predominately of northern European ancestry. Moreover, there is a higher proportion of healthcare workers and correspondingly higher education level in this population, which might affect pattern of healthcare utilisation and disease detection.

\section{Conclusions}

Increased incidence of CVD among patients with sarcoidosis was demonstrated in this population-based cohort, even after controlling for baseline traditional atherosclerotic risk factors.

\section{Acknowledgements}

Author contributions: All authors had a role in conducting the research and writing the manuscript. The final version of this manuscript was approved by all authors.

\section{References}

1 Heidenreich PA, Trogdon JG, Khavjou OA, et al. Forecasting the future of cardiovascular disease in the United States: a policy statement from the American Heart Association. Circulation 2011; 123: 933-944.

2 Berry JD, Dyer A, Cai X, et al. Lifetime risks of cardiovascular disease. N Engl J Med 2012; 366: 321-329.

3 Maradit-Kremers H, Nicola PJ, Crowson CS, et al. Cardiovascular death in rheumatoid arthritis: a populationbased study. Arthritis Rheum 2005; 52: 722-732.

4 Bessant R, Hingorani A, Patel L, et al. Risk of coronary heart disease and stroke in a large British cohort of patients with systemic lupus erythematosus. Rheumatology 2004; 43: 924-929.

5 Ungprasert P, Suksaranjit P, Spanuchart I, et al. Risk of coronary artery disease in patients with idiopathic inflammatory myopathies: a systematic review and meta-analysis of observational studies. Semin Arthritis Rheum 2014; 44: 63-67.

6 Libby P. Inflammation in atherosclerosis. Nature 2012; 420: 868-874.

7 Montecucco F, Mach F. Common inflammatory mediators orchestrate pathophysiological processes in rheumatoid arthritis and atherosclerosis. Rheumatology 2009; 48: 11-22.

8 Rybicki BA, Major M, Popovich J Jr, et al. Racial differences in sarcoidosis incidence: a 5-year study in a health maintenance organization. Am J Epidemiol 1997; 145: 234-241.

9 Cozier YC, Berman JS, Palmer JR, et al. Sarcoidosis in black women in the United States: data from the Black Women's Health Study. Chest 2011; 139: 144-150.

10 Zöller B, Li X, Sundquist J, et al. Risk of subsequent coronary heart disease in patients hospitalized for immune-mediated diseases: a nationwide follow-up study from Sweden. PLoS One 2012; 7: e33442.

11 Rocca WA, Yawn BP, St Sauver JL, et al. History of the Rochester Epidemiology Project: half a century of medical records linkage in a U.S. population. Mayo Clin Proc 2012; 87: 1202-1213.

12 Ungprasert P, Carmona EM, Utz JP, et al. Epidemiology of sarcoidosis 1946-2013: a population-based study. Mayo Clin Proc 2016; 91: 183-188.

13 Costabel U, Hunninghake GW. ATS/ERS/WASOG statement on sarcoidosis. Sarcoidosis Statement Committee. American Thoracic Society. European Respiratory Society. World Association for Sarcoidosis and Other Granulomatous Disorders. Eur Respir J 1999; 14: 735-737.

14 Ho KLK, Anderson KM, Kannel WB, et al. Survival after the onset of congestive heart failure in Framingham Heart Study subjects. Circulation 1993; 88: 107-115.

15 Muir RL. Peripheral arterial disease: pathophysiology, risk factors, diagnosis, and prevention. J Vasc Nurs 2009; 27: 26-30.

16 Moxon JV, Parr A, Emeto TI, et al. Diagnosis and monitoring of abdominal aortic aneurysm: current status and future prospects. Curr Probl Cardiol 2010; 35: 512-548.

17 D'Agostino RB Sr, Vasan RS, Pencina MJ, et al. General cardiovascular risk profile for use in primary care: the Framingham Heart Study. Circulation 2008; 117: 743-753. 
18 Gooley TA, Leisenring W, Crowley J, et al. Estimation of failure probabilities in the presence of competing risks: new representations of old estimators. Stat Med 1999; 18: 695-706.

19 Lee YH, Choi SJ, Ji JD, et al. Overall and cause-specific mortality in systemic lupus erythematosus: an update meta-analysis. Lupus 2016; 25: 727-734.

20 Cybulsky MI, Iiyama K, Li H, et al. A major role for VCAM-1, but not ICAM-1, in early atherosclerosis. J Clin Invest 2001; 107: 1255-1262.

21 Rajavashisth TB, Liao JK, Galis ZS, et al. Inflammatory cytokines and oxidized low density lipoproteins increase endothelial cell expression of membrane type 1-matrix metalloproteinase. J Biol Chem 1999; 274: 11924-11929.

$22 \mathrm{Xu}$ J, Lupu F, Esmon CT. Inflammation, innate immunity and blood coagulation. Hamostaseologie 2010; 30: 5-6.

23 Riva N, Donadini MP, Ageno W. Epidemiology and pathophysiology of venous thromboembolism: similarities with atherothrombosis and the role of inflammation. Thromb Haemost 2015; 113: 1176-1183.

24 Kurko J, Besenyei T, Laki J, et al. Genetics of rheumatoid arthritis - a comprehensive review. Clin Rev Allergy Immunol 2013; 45: 170-179.

25 Paakkanen R, Lokki ML, Seppanen M, et al. Proinflammatory $H L A-D R B 1^{\star} 01$-haplotype predisposes to ST-elevation myocardial infarction. Atherosclerosis 2012; 221: 461-466.

26 Crowson CS, Liao KP, Davis JM 3rd, et al. Rheumatoid arthritis and cardiovascular disease. Am Heart J 2013. 166: 622-628.

27 Rossman MD, Thompson B, Federick M, et al. HLA-DRB1*1101: a significant risk factor for sarcoidosis in blacks and whites. Am J Hum Genet 2003; 73: 720-735.

28 Maradit Kremers H, Reinalda MS, Crowson CS, et al. Glucocorticoids and cardiovascular and cerebrovascular events in polymyalgia rheumatica. Arthritis Rheum 2007; 57: 279-286. 